\title{
REPLICANDO O EFEITO TAKETE-MALUMA EM PORTUGUÊS BRASILEIRO
}

\section{THE REPLICATION OF TAKETE-MALUMA EFFECT IN BRAZILIAN PORTUGUESE}

\author{
Mahayana Cristina Godoy ${ }^{1}$ \\ Ana Clarissa Viana Duarte ${ }^{2}$ \\ Fernando Laerty Ferreira da Silva ${ }^{3}$ \\ Gabriela Fernandes Albano ${ }^{4}$ \\ Rayara Jayne Pereira de Souza ${ }^{5}$ \\ Youssef Ud Alighiere de Paiva Macena da Silva ${ }^{6}$ \\ Universidade Federal do Rio Grande do Norte
}

\section{RESUMO}

Este artigo tem como objetivo discutir o papel da iconicidade como fenômeno linguístico e trazer evidências empíricas sobre a necessidade dessa discussão. Para tanto, investigamos se falantes de Português Brasileiro (PB) constroem as mesmas relações entre fonemas e categorias perceptuais que falantes de outras línguas, um fenômeno descrito como simbolismo sonoro (LOCKWOOD e DINGEMANSE, 2015). Isso foi feito por meio da replicação do experimento Takete-Maluma (KÖHLER, 1929) e uma variação desse teste que tem como objetivo avaliar o papel das consoantes e das vogais na construção do simbolismo sonoro (NIELSEN e RENDALL, 2011). Nossos resultados revelam que falantes nativos de PB fazem a mesma relação entre som e formas geométricas que falantes de outras línguas. Com esses resultados, nosso artigo ressalta a necessidade de integrarmos a iconicidade em modelos que pretendam dar conta do fenômeno da linguagem e a importância de mais estudos experimentais sobre simbolismo sonoro no PB.

PALAVRAS-CHAVE: iconicidade; simbolismo sonoro; takete-maluma; correspondência entre modalidades; psicolinguística.

\begin{abstract}
In this paper we discuss the role of iconicity in language and show empirical evidence suggesting why this discussion is needed. In order to do so, we investigate whether Brazilian Portuguese (BP) speakers show the same mapping between phonemes and perceptual categories as speakers of other languages, a phenomenon called sound symbolism (LOCKWOOD and DINGEMANSE, 2015). This was done by replicating the Takete-Maluma experiment (KÖHLER, 1929) and a variation of this test aimed at assessing the role that consonants and

\footnotetext{
${ }^{1}$ Laboratório de Estudos Experimentais em Linguagem (LEElin/UFRN); Programa de Pós-graduação em Estudos da Linguagem (PPgEL/UFRN). Email: mgodoy@cchla.ufrn.br

2 Mestranda em Estudos da Linguagem pelo Programa de Pós-graduação em Estudos da Linguagem (PPgEL) da Universidade Federal do Rio Grande do Norte (UFRN). Email: vianinha_anita@hotmail.com.

${ }_{3}^{3}$ Mestrando em Estudos da Linguagem pelo Programa de Pós-graduação em Estudos da Linguagem (PPgEL) da Universidade Federal do Rio Grande do Norte (UFRN).Email: f.laerty@hotmail.com.

4 Mestre em Estudos da Linguagem, atualmente doutoranda pelo Programa de Pós-graduação em Estudos da Linguagem (PPgEL) da Universidade Federal do Rio Grande do Norte (UFRN) e bolsista da CAPES. Email: gabriela_falbano@hotmail.com.

${ }_{5}^{5}$ Mestranda em Estudos da Linguagem pelo Programa de Pós-graduação em Estudos da Linguagem (PPgEL) da Universidade Federal do Rio Grande do Norte (UFRN).Email: rayara.souza06@gamail.com.

${ }_{6}^{6}$ Mestrando em Estudos da Linguagem pelo Programa de Pós-graduação em Estudos da Linguagem (PPgEL) da Universidade Federal do Rio Grande do Norte (UFRN). Email: ud.alighiere@hotmail.com.
} 
vowels play in sound symbolism (NIELSEN and RENDALL, 2011). Our results show that BP speakers make the same associations between sounds and geometric shapes as speakers of other languages. We stress the importance of incorporating iconicity into models that account for linguistic phenomena and the need of future experimental studies on sound symbolism in BP.

KEY-WORDS: iconicity; sound symbolism; takete-maluma; cross-modal correspondence; psycholinguistics

\section{INTRODUÇÃO}

Dentre os temas recorrentes nos estudos da linguagem, um dos mais antigos diz respeito à discussão sobre quão arbitrárias ou icônicas são as palavras e construções que utilizamos. No Crátilo de Platão, essa discussão se faz presente no diálogo entre Hermógenes e Sócrates, no qual defendem posições antagônicas acerca da questão. Enquanto aquele defende a ideia de que a língua seria um sistema de convenções, este sugere uma ligação natural entre a estrutura de uma palavra e o objeto por ela denotado no mundo. No início do século XX, a questão ganha novo fôlego após a publicação do Curso de linguística geral, na qual Saussure define a arbitrariedade como um dos princípios do signo linguístico. Desde então, a arbitrariedade é vista como um dos elementos definidores da língua, e essa posição é, até hoje, pouco disputada dentro de determinadas correntes teóricas de cunho formal.

Contudo, ainda que com menor destaque, o princípio da arbitrariedade foi disputado durante o século XX. Há oitenta e sete anos, em um dos experimentos mais replicados sobre o tema, Köhler (1929) reportou que certos sons são sistematicamente associados a determinadas formas geométricas, questionando a suposta arbitrariedade entre som e significado. Na segunda metade do século passado, a iconicidade passa a constituir tema profícuo nos estudos funcionalistas. A ideia de que a organização linguística está sujeita a pressões do uso e a necessidades comunicativas abre a possibilidade de investigar como o funcionamento morfológico e sintático das línguas obedece a "propriedades de conceitualização humana do mundo e o funcionamento da mente" (FURTADO DA CUNHA e TAVARES, 2007, p. 21).

O campo da psicolinguística tem testemunhado um crescimento no número de estudos sobre aspectos icônicos da linguagem, causado, principalmente, pelo acúmulo de evidências empíricas de que a iconicidade desempenha papel relevante em pelo menos alguns aspectos do processamento (NYGAARD, COOK e NAMY, 2009; WESTBURY, 2005) e da aquisição e desenvolvimento da linguagem (ORTEGA, SÜMER e ÖZYÛREK, 2016; IMAI, KITA, NAGUMO e OKADA, 2008). Como resultado, alguns trabalhos têm defendido que a dicotomia arbitrário/icônico é ultrapassada, e que um modelo adequado sobre a língua tem como desafio entender como arbitrariedade e iconicidade atuam de forma conjunta e decisiva na compreensão e na produção linguística, tornando a língua um sistema complexo e eficiente.

Um exemplo dessa tentativa de conciliação é o trabalho de Lockwood e Dingemanse (2015), que discute como a arbitrariedade e a iconicidade na língua têm um papel decisivo na comunicação. Segundo lembram os autores, a arbitrariedade possibilita a combinação de símbolos de forma ilimitada, aumentando, do ponto de vista comunicativo, a variedade de conceitos e relações a serem expressas. Nesse sentido, conclui-se que um sistema somente baseado na iconicidade seria prejudicial à nossa capacidade de expressão de conceitos específicos, tendo em vista que isso imporia limitações quanto às distinções conceituais que poderíamos fazer. Contudo, Lockwood e Dingemanse (2015) ainda salientam que um sistema linguístico apenas arbitrário causaria dificuldade de aprendizagem, visto que não haveria ligação entre forma e experiência humana. De fato, novos trabalhos indicam que a aquisição da linguagem tende a apoiar-se em relações icônicas quando possível, e que relações menos icônicas seriam aprendidas tardiamente (ORTEGA, SÜMER e ÖZYÛREK, 2016), possivelmente porque relações mais 
icônicas se baseiam em sistemas cognitivo-perceptuais já existentes (CUSKLEY e KIRBY, 2013). Para Lockwood e Dingemanse (2015), portanto, arbitrariedade e iconicidade devem ser encaradas não como conceitos que propõem visões diferentes de língua, mas como conceitos complementares, uma vez que os benefícios de um parecem ter por objetivo compensar as vulnerabilidades do outro.

Seguindo a proposta conciliadora dos autores, o presente artigo busca contribuir para a investigação do papel da iconicidade na organização do sistema linguístico. Através da replicação do experimento, Takete-Maluma, proposto por Köhler (1929), e de uma variação deste focando na distinção entre simbolismo sonoro de vogais e consoantes (NIELSEN e RENDALL, 2011), observamos se falantes do Português Brasileiro (PB) constroem as mesmas relações entre fonemas e categorias perceptuais que falantes de outras línguas. Esse estudo torna-se relevante no momento em que estudos recentes começam a discutir a universalidade do fenômeno descrito por Köhler e propõem condições em que o mapeamento som/significado pode ou não ser observado (STYLES e GAWNE, 2017). Além da óbvia contribuição para os estudos sobre simbolismo sonoro, que têm crescido desde a virada do século, nosso estudo traz duas contribuições inéditas para o estudo da iconicidade em PB.

Em primeiro lugar, exploramos um fenômeno pouco estudado na língua em questão: a iconicidade sonora. Ainda que haja alguns trabalhos sobre o uso de ideofones na língua (CRUZ, 2000; CRUZ e FERNANDES, 2004), desconhecemos estudo sistemático sobre simbolismo sonoro nos estudos linguísticos do PB. Além disso, conduzimos nossa pesquisa a partir de metodologia experimental, um expediente comum à psicolinguística, mas pouco empregada nos estudos de linguística teórica e descritiva. Acreditamos que uma metodologia experimental pode trazer maior compreensão sobre os fenômenos linguísticos, especialmente em quadros teóricos que tratam a língua como fenômeno natural, dinâmico, sujeito a pressões de uso (JAEGER e TILY, 2011). Assim, ao investigarmos a questão de forma experimental e tratarmos das implicações de nossos achados à luz de estudos psicolinguísticos, buscamos construir maior sinergia entre as áreas teórico-descritiva e teórico-experimental na descrição do uso do PB.

O restante deste artigo organiza-se da seguinte maneira: na próxima seção, fazemos uma revisão sobre o simbolismo sonoro, discutindo os achados empíricos da área e o suposto papel que esse fenômeno desempenha na aquisição da linguagem. Depois, descrevemos com mais detalhes o experimento de Nielsen e Rendall (2011), o qual replicamos em nosso trabalho. Por fim, apresentaremos os métodos e resultados de nosso trabalho, seguidos das nossas conclusões.

\section{Os estudos sobre simbolismo sonoro}

Há, hoje, um número volumoso de pesquisas sobre a iconicidade no $\mathrm{PB}$, especialmente a partir da perspectiva do funcionalismo. Nessa perspectiva, os fenômenos linguísticos são explicados através de princípios, propriedades e motivações de natureza cognitivo-comunicativa e cultural. Na concepção de Givón (1995), o princípio da iconicidade refere-se à correlação motivada entre forma e função, não só no nível sintático, mas no morfológico e no morfossintático. Como consequência, os estudos sobre iconicidade no PB focam na relação entre forma e função em níveis linguísticos como a morfologia (CUNHA, OLIVEIRA e MARTELOTTA, 2003; OLIVEIRA e CEZARIO, 2007) ou a sintaxe (BRAGA, 1996; MOLLICA, 1998), e poucos se detêm em questões relacionadas à iconicidade associada a sons específicos.

A própria definição de iconicidade sonora parece abarcar uma variedade de fenômenos distintos, tais como a imitação de sons, ao estilo das onomatopéias, a ocorrência de ideofones, itens lexicalizados que evocam uma imagem sensória e a presença de fonastemas, sequências recorrentes que não chegam a formar um morfema contrastivo, mas que indicam relação entre som e significado (e.g., o par gl-, em inglês, recorrente em vocábulos relacionados a brilho, 
luminosidade e visão, como glimmer, glitter, glare, glisten). De modo geral, o que todos esses fenômenos têm em comum é uma relação entre som e percepções sensório-motoras que não se limitariam à forma, mas remeteriam também a categorias como cor, sabor e luminosidade.

Um estudo pioneiro a investigar essa relação entre forma e som foi desenvolvido por Köhler $(1929,1947)$ e ficou conhecido como o experimento Takete-Maluma. Os resultados desse experimento, que descreveremos com mais detalhes adiante, indicam que fonemas como /o/, $/ \mathrm{m} / \mathrm{e} / \mathrm{l} / \mathrm{seriam}$ preferencialmente utilizados para fazer referência a formas arredondas, enquanto formas pontudas seriam preferencialmente nomeadas com palavras que continham fonemas como $/ \mathrm{e} /, / \mathrm{k} / \mathrm{e} / \mathrm{t} / \mathrm{Na}$ esteira desse experimento, outras pesquisas replicaram os mapeamentos reportados pelo autor (HOLLAND e WERTHEIMER, 1964; RAMACHANDRAN e HUBBARD, 2001; PEIFFER-SMADJA, 2010; FORT, MARTIN e PEPERKAMP, 2015, entre outros) ou encontraram novas relações entre som e significados ligados a percepções sensório-motoras (THOMPSON e ESTES, 2011; OHTAKE e HARYU, 2013; ASANO e YOKASAWA, 2011), abrindo caminho para se discutir a extensão dessas relações e a sua pertinência para o sistema linguístico.

Quanto à extensão do fenômeno, estudos recentes têm mostrado que esse mapeamento entre som e significado ocorre em diversas línguas e perpassa diferentes categorias perceptuais. Em uma extensa revisão de literatura, Lockwood e Dingemanse (2015, p. 6) listam muitos dos achados da área e salientam que os efeitos das consoantes parecem ser mais proeminentes do que as vogais, embora ambas desempenhem um papel no estabelecimento da relação icônica. De modo geral, os achados atuais resumidos pelos autores indicam que (i) consoantes sonoras e vogais posteriores (redondas) são relacionadas à escuridão (HIRATA ET AL., 2011), a formas curvas (KÖHLER, 1929, 1947) e a movimentos lentos (CUSKLEY, 2013), enquanto consoantes não-sonoras e vogais frontais altas foram relacionadas à luminosidade, a formas pontudas e a movimentos rápidos; (ii) vogais baixas e posteriores são associadas a objetos grandes, enquanto vogais altas e frontais são associadas a objetos pequenos (THOMPSON e ESTES, 2011; OHTAKE e HARYU, 2013); (iii) vogais altas estão associadas à vermelhidão, enquanto vogais baixas estão associadas a tons de amarelo (MOOS ET AL., 2014); (iv) vogais mais altas e posteriores são associadas à percepção de um gosto doce, enquanto vogais baixas e mais posteriores são associadas a gostos amargos (SIMNER, CUSKLEY e KIRBY, 2010).

Com relação à pertinência do simbolismo sonoro para o sistema linguístico, os estudos que têm se dedicado à questão apontam para o papel desempenhado pela iconicidade no processo de aquisição da linguagem. Lockwood, Dingemanse e Hagoort (2016) identificaram que a ligação simbólica entre som e significado é capaz de promover com maior facilidade o aprendizado de novas palavras em uma língua estrangeira. Os autores manipularam o ensino de ideofones do japonês para um grupo de falantes de inglês e perceberam que esses falantes aprendiam melhor os ideofones quando eles eram apresentados com seu significado real, comparativamente a uma condição em que os ideofones eram apresentados com o significado oposto. Esse achado vai ao encontro de outros estudos que identificaram que pessoas que não falavam japonês eram capazes de escolher corretamente o significado de um ideofone em uma taxa superior ao que ocorreria ao acaso (KITA, 1997; AKITA 2009).

As pesquisas sobre simbolismo sonoro não se mostraram produtivas apenas no tocante à aprendizagem de um segundo idioma, mas também na investigação da relação som-significado para a aquisição da primeira língua por crianças. Em um estudo de caso, Elsen (1991; apud LOCKWOOD e DINGEMANSE, 2011) investigou os dados de aquisição da linguagem de uma criança alemã, Annalena, e documentou que, aos 11 meses, ela fazia uso extensivo de formas onomatopeicas, chegando a quase $40 \%$ do seu vocabulário. Conforme desenvolveu suas habilidades fonológicas, a criança foi sistematicamente trocando as formas onomatopaicas por palavras convencionais do seu idioma, substituindo, por último, aquelas onomatopeias cuja palavra correspondente era fonologicamente mais complexa. Esse estudo vai ao encontro de 
trabalhos mais recentes sobre a aquisição de língua de sinais, que indicam que crianças tendem a adquirir inicialmente sinais mais icônicos (THOMPSON, VINSON, WOLL e VIGLIOCCO, 2012) e que a sinalização de adultos, quando dirigida a crianças, faria maior uso de sinais icônicos comparativamente à sinalização dirigida a outros adultos (ORTEGA, SÜMER e ÖZYÛREK, 2016). Similarmente, o uso de ideofones para descrição de movimentos tende a ser mais comum em crianças que em adultos, e a coocorrência de gestos icônicos para acompanhar a produção dessas palavras é maior entre crianças (KITA, ÖZYÛREK, ALLEN, ISHIKUZA, 2010). Desse modo, há evidência para acreditarmos, com outros autores (IMAI e KITA, 2014; ORTEGA, SÜMER e ÖZYÛREK, 2016), que a iconicidade, incluindo aí as representações icônicas no campo do simbolismo sonoro, é usada como boostrap no estabelecimento de representações lexicais durante a aquisição da linguagem. O estudo das relações icônicas passa a ser, então, importante para uma compreensão efetiva do funcionamento da linguagem.

Apesar do crescente interesse no simbolismo sonoro observado nos últimos anos, no Brasil há poucos trabalhos que investigam esse tópico. Estudos sobre a relação entre som e iconicidade no PB têm se concentrado na caracterização dos ideofones em diferentes dialetos dessa língua (CRUZ, 2000; MELO 2007; FERNANDES e CRUZ, 2011), identificando algumas de suas características e usos, como a preferência pelo uso de bilabiais na construção desses itens lexicais e a sua maior ocorrência como designador, intensificador e marcador conversacional (CRUZ e FERNANDES, 2004). Contudo, pouco sabemos sobre como os falantes nativos de português brasileiro se comportam frente a simbolismos sonoros que têm sido caracterizados como perenes a despeito de diferentes tipologias linguísticas, isto é, não sabemos se falantes de PB fazem os mesmos mapeamentos entre sons e categorias perceptuais que são descritos para outras línguas. Como nosso trabalho tem por objetivo preencher esta lacuna por meio da replicação de um experimento de simbolismo sonoro com falantes de $\mathrm{PB}$, descreveremos inicialmente os detalhes do experimento original.

\section{0 experimento takete-maluma}

Como já descrevemos brevemente, o fenômeno Takete-Maluma, reportado inicialmente por Köhler $(1929,1947)$, é fruto de um estudo experimental que buscou investigar a relação entre forma-som. Nesse experimento, os participantes eram convidados a associar formas curvas ou pontudas a não-palavras. Essas não-palavras eram criadas a partir da combinação de vogais e consoantes pré-determinadas, e o objetivo principal do experimento era averiguar se determinados fonemas eram preferencialmente associadas a uma ou à outra forma. Os resultados obtidos por esse experimento mostraram que há uma associação entre formas redondas e nãopalavras como maluma e formas pontudas e não-palavras como takete.

Embora Köhler $(1929,1947)$ tenha obtido resultados que confirmassem a relação entre palavras inexistentes com determinadas formas, uma série de questionamentos foram levantados a respeito de seus resultados. A principal delas diz respeito ao papel das consoantes e das vogais ao determinar a relação entre forma e som. Alguns estudos identificaram que vogais como /o/ são associadas a formas elípticas, enquanto vogais como /e/ são associadas a triângulos para falantes de inglês e tcheco (TARTE e BARRITT, 1971; TARTE, 1974), o que poderia explicar o efeito Takete-Maluma. Contudo, outros estudos identificaram que elementos pontudos são associados a oclusivas como $/ \mathrm{k} / \mathrm{e} / \mathrm{t} /$, ao passo que figuras redondas são preferencialmente associadas a consoantes como $/ 1 / \mathrm{e} / \mathrm{m} /$ (WESTBURY, 2005), o que também seria capaz de explicar o efeito documentado por Köhler.

Para investigar essa questão, Nielsen e Rendall (2011) replicaram o estudo do fenômeno Takete-Maluma, mas inserindo em seu experimento uma segunda condição em que os pares de vogais e consoantes eram trocados entre as palavras usadas, resultando em itens como Maleme e Takuta. Os resultados mostraram que, nas situações testadas, o mapeamento de som e de forma 
foi feito a partir das consoantes, ou seja, palavras como Maleme foram associadas a formas redondas, enquanto formas pontiagudas eram referidas por palavras como Takuta. Segundo os autores, esses resultados indicam que, no experimento de Köhler, seriam as consoantes, mais do que as vogais, os elementos mais proeminentes no estabelecimento do simbolismo sonoro. Posteriormente, o papel desempenhado pelas consoantes no estabelecimento do mapeamento entre os sons e as formas em questão foi confirmado por outros experimentos (FORT, MARTIN e PEPERKAMP, 2015).

Neste artigo, apresentamos uma replicação do experimento Takete-Maluma de forma similar ao conduzido por Nielsen e Rendall (2011) para testar o papel das consoantes e das vogais no simbolismo sonoro para falantes de PB. Convém mencionar que nossa hipótese inicial seria de que o achado mais geral associado a palavras como Takete e Maluma seria replicado na língua, uma vez que há apenas dois casos reportados de falha na replicação no fenômeno ${ }^{7}$. Contudo, por haver comparativamente menos estudos sobre o papel da consoante e da vogal na tarefa proposta, aliado ao fato de não haver qualquer estudo que conheçamos sobre os simbolismos sonoros no PB, não é possível saber com certeza se os achados de Nielsen e Rendall (2011) sobre as consoantes replicariam para os falantes do PB. Em outras palavras, não é possível saber se o mapeamento entre som e forma seria determinado primordialmente pelas consoantes.

\subsection{Materiais e métodos}

Para replicarmos o experimento de Nielsen e Rendall (2011), apresentamos aos participantes uma tarefa de nomeação de escolha-forçada como a realizada pelos autores. Aos participantes eram mostradas duas imagens de formas abstratas, uma pontuda e uma mais arredondada. Em nosso experimento, essas imagens eram apresentadas impressas em uma folha de papel ${ }^{8}$. Nessa mesma folha eram apresentadas, em letras maiúsculas, duas não-palavras, e o participante tinha que ligar, com um lápis, qual das palavras nomeava melhor as figuras expostas (cf. Figura 1). Como havia duas imagens e duas palavras, ao escolher uma das palavras para nomear uma figura, o participante forçosamente definia que a outra palavra deveria nomear a outra figura, o que caracteriza a tarefa de escolha-forçada. Somente após decidirem sobre a nomeação, os participantes recebiam do pesquisador outra folha de papel com outro par de imagens e outro par de palavras para continuar o experimento. O participante não poderia voltar para mudar seu julgamento sobre os itens anteriores.

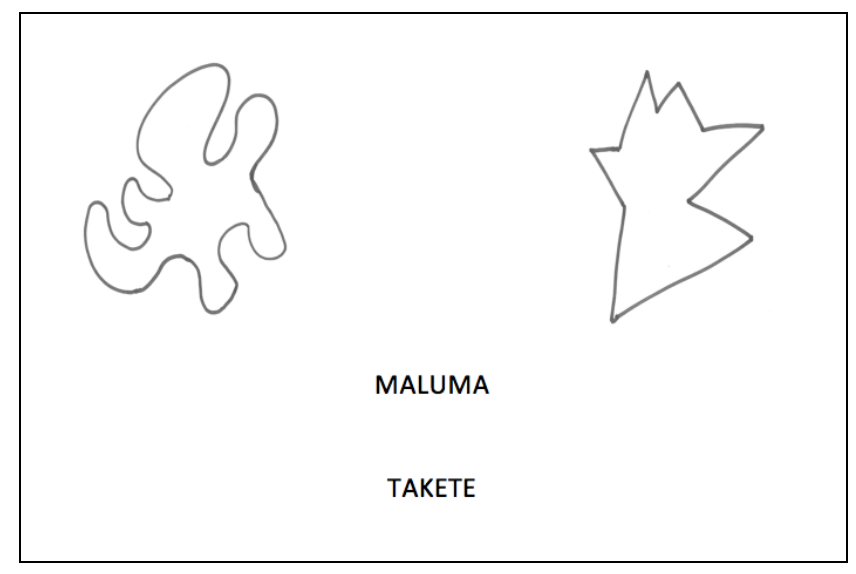

\footnotetext{
7 As línguas para as quais não foi encontrado o efeito Takete-Maluma são um dialeto do Hunjara, falado na PapuaNova Guiné pela população Songe (ROGERS e ROSS, 1975), e um dialeto do Yolmo, o Syuba, falado no Nepal (STYLES e GAWNE, 2017).

${ }^{8}$ Nesse aspecto, nosso experimento difere daquele de Nielsen e Rendall (2011), que apresentaram o estímulo por meio de um computador.
}

Revista do GELNE, Natal/RN, Vol. 20 - Número 1: p. 87-100. 2018 
Figura 1: Exemplo de material apresentado ao participante

Para os pares de imagens, criamos versões semelhantes aos 5 pares de imagens utilizadas por Nielsen e Rendall (2011), que, por sua vez, eram adaptadas das imagens de Köhler (1929) e de Maurer, Pathman e Mondloch (2006) (cf. Tabela 1).

Par de imagens 1

Tab. 1: Pares de figuras utilizados no experimento

Os pares de palavras na Condição 1 também foram os mesmos utilizados por Nielsen e Rendall (2011). Os pares descritos como Condição 1 haviam sido emprestados de Köhler (1929, 1947) e Maurer et al. (2006), e consistiam de palavras em que vogais sonoras como /m/, /g/ e /l/ coocorriam com a vogais arredondadas como /o/ ou /u/, enquanto vogais oclusivas e nãosonoras como $/ \mathrm{p} / \mathrm{h} / \mathrm{t} / \mathrm{e} / \mathrm{k} /$ eram acompanhadas da vogal não-arredondada /e/. Os pares descritos na Condição 2 foram criados por Nielsen e Rendall (2011). Nessa condição, houve troca de vogais entre os pares de palavras, de modo a poder testar o efeito da vogal e da consoante separadamente. A Tabela 2 mostra os pares de palavras usados. Entre parênteses, mostra-se a indicação de que a palavra foi mais facilmente associada a uma imagem redonda ( $r$ ) ou pontuda (p) de acordo com os resultados de Nielsen e Rendall (2011).

\begin{tabular}{|ll|ll|}
\hline \multicolumn{2}{|c|}{ Condição 1 } & \multicolumn{2}{c|}{ Condição 2 } \\
\hline KUHTAY $(\mathrm{p})$ & BAAMOO $(\mathrm{r})$ & BUHMAY $(\mathrm{r})$ & KAATOO $(\mathrm{p})$ \\
\hline TUHKEETEE $(\mathrm{p})$ & MAABOOMAA $(\mathrm{r})$ & MUHBEEMEE $(\mathrm{r})$ & TAAKOOTAA $(\mathrm{p})$ \\
\hline
\end{tabular}




\begin{tabular}{|ll|ll|}
\hline KAYKEE $(\mathrm{p})$ & BOOBAA $(\mathrm{r})$ & BAYBEE $(\mathrm{r})$ & KOOKAA $(\mathrm{p})$ \\
\hline TEETAY $(\mathrm{p})$ & GOGAA $(\mathrm{r})$ & GEEGAY $(\mathrm{r})$ & TOTAA $(\mathrm{p})$ \\
\hline TAKETE $(\mathrm{p})$ & MALUMA $(\mathrm{r})$ & MALEME $(\mathrm{r})$ & TAKUTA $(\mathrm{p})$ \\
\hline
\end{tabular}

Tabela 2: Palavras usadas para nomeação de palavras e indicação de preferência por imagens pontudas (p) ou redondas (r) de acordo com Nielsen e Rendall (2011)

Convém notar uma distinção entre o PB e o inglês, língua do qual os itens na Tabela 2 foram emprestados. O fonema representado ortograficamente pelo grafema/uh/ é distinto nas duas línguas, sendo uma vogal arredondada em PB e uma vogal não arredondada em inglês. Como se trata da primeira replicação desse experimento para o $\mathrm{PB}$, decidimos fazer o mínimo de alterações possíveis, e mantivemos a ocorrência do grafema /uh/ para as palavras que, em tese, teriam as vogais não-arredondadas. Além disso, uma vez que o PB replique o observado por Nielsen e Rendall (2011), a presença de vogais de diferentes tipos não alteraria o padrão de mapeamento entre consoantes e formais arredondadas e pontudas.

A apresentação dos pares de imagens com os pares de palavras foi aleatorizada em 5 listas diferentes, de modo que o mesmo par de imagens ocorria com um par de palavras diferente em cada uma das listas. A ordem de apresentação do par de palavras e de imagens no papel também foi aleatorizada, de modo a evitar efeitos resultantes da disposição desses elementos no papel.

Cada participante viu o mesmo par de imagens duas vezes: uma vez na Condição 1, outra na Condição 2. Contudo, para um mesmo participante, o par de imagens nunca era apresentado pela segunda vez com o par de palavras similar ao primeiro; isto é, o participante que visse o par de imagens 1 associado a TAKETE/MALUMA na Condição 1, não o via associado ao par TAKUTA/MALEME na Condição 2. A ordem de apresentação das condições (Condição 1/Condição 2) também foi aleatorizada entre os participantes.

Participaram voluntariamente do experimento 24 falantes nativos de $\mathrm{PB}, 14$ do sexo feminino, com idades entre 21 e 52 anos (média $=29,5$; desvio-padrão $=10,5$ ).

\subsection{Análise de dados e resultados}

Os dados foram tabelados considerando como variável dependente sua conformidade com a hipótese estabelecida por Nielsen e Rendall (2011). Portanto, nossa variável resposta era categórica e tinha dois níveis: ou a resposta dada pelo participante se conformava à hipótese de Nielsen e Rendall, ou era contrária a essa hipótese. Para a Condição 1 conformar-se à hipótese, implicava que as palavras com vogais arredondadas e consoantes sonoras fossem associadas às imagens redondas. Para a Condição 2 conformar-se à hipótese, implicava que as palavras com consoantes sonoras fossem associadas às imagens redondas, mesmo que tais consoantes viessem acompanhadas de vogais não-arredondadas. Em não havendo diferenças entre as respostas obtidas para Condição 1 e para Condição 2, saberíamos que qualquer efeito observado seria resultado da manipulação da consoante.

A fim de analisar os dados, utilizamos um modelo de regressão logística misto com o pacote lme4 no software estatístico R. Nosso modelo tinha por objetivo identificar se, para a Condição 1 e para a Condição 2, a resposta dos participantes se conformava à hipótese de Nielsen e Rendall (2011) em uma taxa que não pudesse ser atribuída ao acaso (i.e., chance maior de $50 \%$ de atribuir palavras com determinados fonemas a imagens pontudas ou redondas) e se havia diferença de conformidade à hipótese entre as duas condições.

Primeiramente, investigamos se a manipulação da Condição influenciou nas taxas de respostas dos participantes. Para tanto, nosso modelo tinha como variável resposta a resposta dos participantes em sua relação com a hipótese assumida (i.e., uma variável de dois níveis: "conforma-se à hipótese" ou "não se conforma à hipótese), e como variável preditora a Condição (1 ou 2). Também inserimos interceptos aleatórios por itens e por participantes. Através da 
comparação de modelos aninhados (BAAYEN, DAVIDSON e BATES, 2008), identificamos que a variável Condição não contribui significativamente para o modelo $\left(\chi^{2}(1)=2.3868, \mathrm{p}=\right.$ $0.1224)$.

Com relação à hipótese de que os participantes escolheriam os nomes das figuras em conformidade com a hipótese de Nielsen e Rendall (2011), conduzimos análises separadas para os dados de Condição 1 e de Condição 2, ajustando um modelo de regressão logística apenas com uma constante como preditora e com interceptos para participante e item. Os dados indicam que os participantes nomearam as figuras de acordo com as hipóteses em uma taxa superior a $50 \%$ na Condição $1(\beta=1.3626, \mathrm{z}=4.471, \mathrm{p}<0.0001)$ e na Condição $2(\beta=1.179, \mathrm{z}=2.741, \mathrm{p}$ $=0.00613$ ). Isso pode ser visto na Fig. 2 , que apresenta graficamente os dados previstos com intervalo de confiança de $95 \%$.

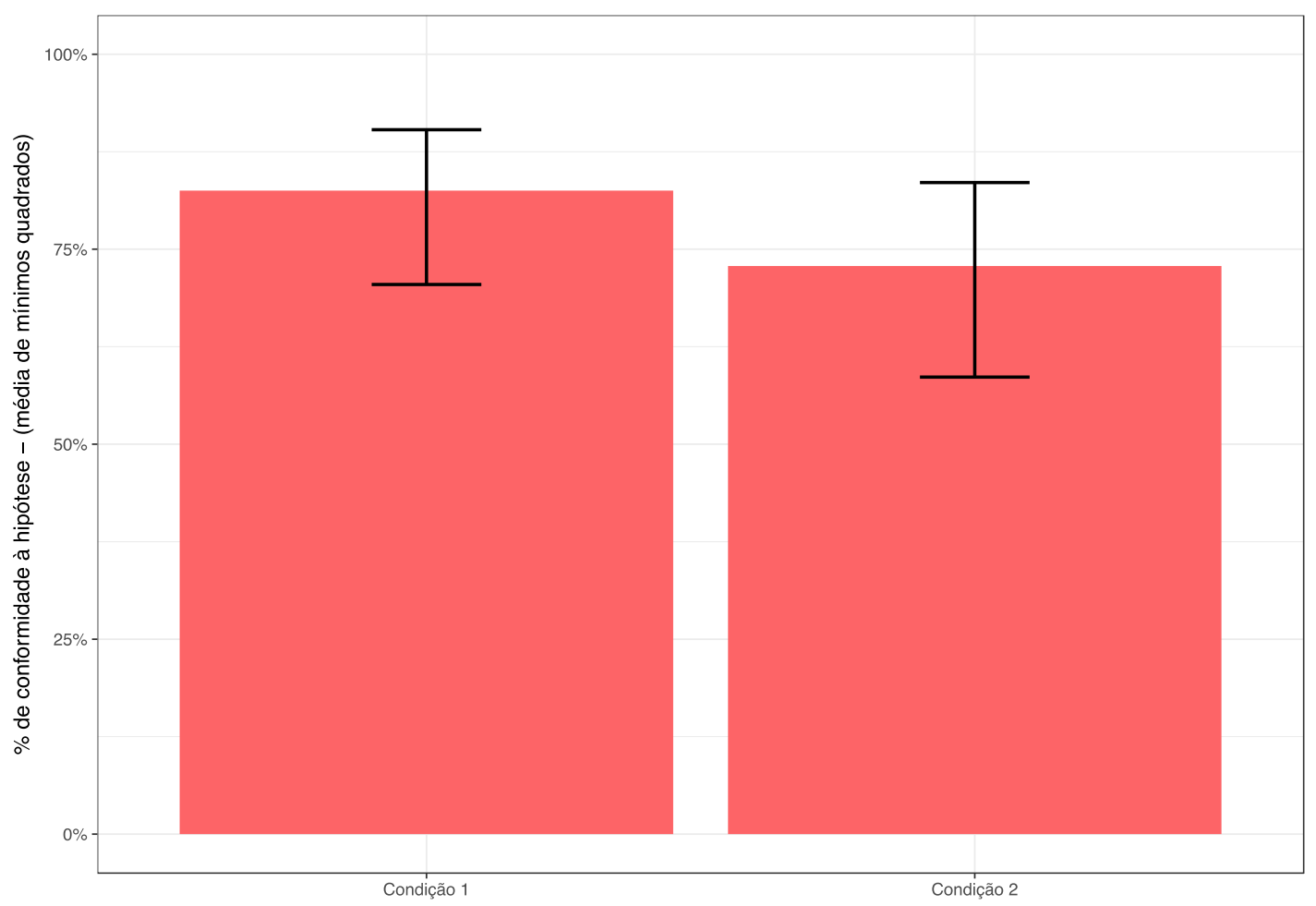

Figura 2: Efeito do tipo de Condição na conformidade à hipótese de Nielsen e Rendall (2011)

\section{Discussão dos resultados}

Para a condição 1, que foi incluída no experimento de Nielsen e Rendall (2011) para replicar o experimento Takete-Maluma de Köhler (1929, 1947), a hipótese inicial era a de que consoantes sonoras como $/ \mathrm{m} /, / \mathrm{l} / \mathrm{e} / \mathrm{b} / \mathrm{e}$ vogais arredondadas como $/ \mathrm{o} / \mathrm{e} / \mathrm{u} /$ corresponderiam a formas curvas, enquanto consoantes como $/ \mathrm{t} /, / \mathrm{k} / \mathrm{e} / \mathrm{p} /$, e vogais nãoarredondadas corresponderiam a formas pontudas. A porcentagem de conformidade à hipótese para a Condição 1 corrobora essa expectativa, visto que esse índice contabiliza resultados acima de $75 \%$, e o intervalo de confiança não engloba a média de $50 \%$, o que poderia indicar que os resultados poderiam se dar ao acaso. Portanto, falantes de PB apresentam os mesmos padrões de mapeamento entre som e categorias perceptuais que falantes de outras línguas.

A condição 2, por sua vez, teve como objetivo separar o papel das vogais e consoantes no mapeamento observado por vários estudos. Essa condição foi proposta por Nielsen e Rendall (2011), com o intuito de mensurar até que ponto as escolhas feitas são baseadas nos sons consonantais. Replicando o estudo dos autores, nossos dados mostram que os participantes foram guiados pelas associações consonantais para determinar o nome de formas curvas e

Revista do GELNE, Natal/RN, Vol. 20 - Número 1: p. 87-100. 2018 
pontudas, uma vez que a alteração das vogais (a troca de Takete-Maluma por Takuta-Maleme, por exemplo) não ocasionou diferença entre as respostas dadas na Condição 1 e na Condição 2. Também na Condição 2 a distribuição do intervalo de confiança nos mostra que a escolha pela nomeação se conformando à hipótese prévia foi superior a $50 \%$.

\section{CONSIDERAÇÕES FINAIS}

De modo geral, nosso trabalho traz evidências concretas de que (a) falantes do PB são guiados por impressões causadas pelos sons em tarefas de nomeação com não-palavras; (b) o mapeamento feito por falantes dessa língua segue padrões documentados para falantes de línguas tipologicamente distintas. Como consequência, argumentamos que um modelo sobre a linguagem que não abarque a iconicidade é incapaz de explicar adequadamente esse fenômeno. Novamente, reiteramos que não se trata de assumir a linguagem como fenômeno essencialmente icônico e, assim, negar qualquer arbitrariedade inerente. Nossa posição é de que o estudo da iconicidade e, nesse campo, especialmente o do simbolismo sonoro - pode trazer contribuições sobre teorias que pretendam informar sobre a organização do sistema linguístico e/ou sobre processos como o de aquisição, de produção e de processamento da linguagem; portanto, tal estudo não deve ser negligenciado pela assunção de que a natureza da linguagem seria, em sua essência, arbitrária.

Se olharmos nossos achados de forma mais específica, encontramos ainda alguns dados que contribuem para questões recentemente levantadas acerca do simbolismo sonoro. Essas questões não foram tratadas nas seções de revisão de literatura e de metodologia para evitarmos tratar como prévias algumas considerações que são, em fato, discussões post-hoc, visto que foram levantadas por trabalhos publicados após a realização de nosso experimento. No entanto, como o estudo apresentado aqui é inicial e exploratório, e tem como objetivo fomentar pesquisas na área tendo o PB como objeto de investigação, convém levantar essas questões para apontar futuras pesquisas.

Um estudo recente destaca que falhas ao replicar o mapeamento entre som e significado reportado no experimento Takete-Maluma em duas línguas reforçam a necessidade de (a) investigar quão abrangente é o fenômeno; (b) determinar que fatores explicam o sucesso ou falha na replicação desse experimento (STYLES e GAWNE, 2017). Em termos de abrangência do fenômeno, Styles e Gawne (2017) reportam o resultado de uma meta-análise dos estudos feitos sobre o fenômeno para estimar que estudos futuros encontrariam entre $84 \%$ e $94 \%$ de convergência para as respostas esperadas em experimentos que usassem os fonemas identificados com formas curvas ou pontudas. Nosso estudo valida a predição das autoras, uma vez que o valor mínimo previsto $(84 \%)$ está no intervalo de confiança dos dados reportados para a Condição 1, que replica mais de perto os trabalhos usados para a estimativa.

Além disso, as autoras preveem que o mapeamento entre som e forma observado nesses estudos depende da adequação fonotática dos itens experimentais à língua testada. Isso explicaria o porquê de não haver replicação do efeito Takete-Maluma em algumas línguas que não têm, em seu inventário fonológico, os fonemas geralmente utilizados na investigação do fenômeno. Essa hipótese também dá conta de explicar a ocorrência do efeito que reportamos em $\mathrm{PB}$, uma vez que essa língua tem, em seu inventário, os fonemas utilizados nos experimentos realizados. Assim, nossa pesquisa traz evidências adicionais que permitem dar suporte à hipótese das autoras. A evidência com falantes de PB é bem-vinda principalmente se considerarmos que o teste nessa língua amplia o escopo de línguas testadas, uma vez que 9 dos 12 experimentos avaliados por Styles e Gawne foram conduzidos com falantes de língua inglesa. A pergunta que permanece, e que merece ser investigada futuramente, diz respeito ao comportamento de falantes de PB quanto ao simbolismo sonoro em tarefas que empreguem fonemas que não constam no inventário fonológico dessa língua. 
Por fim, há de se considerar a influência da representação ortográfica no mapeamento feito pelos participantes. Uma hipótese possível seria de que fonemas associados a formas curvas são ortograficamente representados com formas curvas (e.g., B para /b/, O para /o/) enquanto fonemas associados a formas pontudas são, no experimento, representados ortograficamente por formas pontudas (e.g., $\mathrm{T}$ para $/ \mathrm{t} / \mathrm{K}$ para $/ \mathrm{k} /$ ). Esse ponto já foi levantado por estudos anteriores que apontam algum efeito da ortografia na escolha dos participantes (CUSKLEY, SIMNER e KIRBY, 2017), mas deve ser visto com cautela para explicar o resultado que reportamos aqui, uma vez que, em nossa manipulação, também houve uso de representações ortográficas pontudas que os participantes usaram preferencialmente para nomear formas redondas (M para /m/ ou L para /1/) e uso de grafemas redondos para indicar fonemas que os participantes julgaram nomear formas pontudas (no caso de P para /p/). Além disso, estudos que apresentaram o estímulo de forma auditiva encontraram efeitos semelhantes aos reportados aqui, indicando que o efeito encontrado em diferentes línguas dificilmente pode ser explicado por um mapeamento entre o grafema exposto ao participante e a forma escolhida (cf. Nielsen e Rendall, 2011, experimento 2). Desse modo, acreditamos que uma explicação baseada exclusivamente na forma do grafema não conseguiria explicar nossos dados de maneira satisfatória, principalmente se considerarmos que, recentemente, discute-se que seja justamente a associação entre som e formas específicas que determinem como esses sons são representados grafemicamente (TUROMAN e STYLES, 2017). Um estudo futuro pode investigar a questão apresentando o sinal auditivamente em $\mathrm{PB}$, ou manipulando os grupos participantes de modo a contrastar o julgamento de adultos alfabetizados e não alfabetizados.

Apesar da clara necessidade de futuras pesquisas, o presente estudo é o primeiro a mostrar que relações estabelecidas por simbolismo sonoro em PB impactam a compreensão linguística. Como consequência, esperamos que nossos dados ensejem mais estudos em psicolinguística - seja na área de aquisição, seja nas áreas de compreensão ou produção - que tratem do tema da iconicidade (sonora ou não) no PB.

\section{REFERÊNCIAS BIBLIOGRÁFICAS}

AKITA, Kimi. A Grammar of Sound-Symbolic Words in Japanese: Theoretical Approaches to Iconic and Lexical Properties of Mimetics. 2009. 370 fls. Tese (Doutorado em Filosofia) - Kobe University, 2009.

ASANO, Michiko; IMAI, Mutsumi; KITA, Sotaro; KITAIO, Keiichi; OKADA, Hiroyuki; THIERRY, Guillaume. Sound symbolism scaffolds language development in preverbal infants.

BRAGA, Maria Luiza. Processo de redução: o caso das orações de gerúndio. In: KOCH, Ingedore Villaça (Org.). Gramática do português falado: desenvolvimento. Campinas: Unicamp, 1996. p. 231-251.

CRUZ, Regina. Sound Symbolism in Brazilian Portuguese: a study of ideophones. In: The procedings of the conference gesture: meaning and use. Porto (Portugal): Universidade de Fernando Pessoa, 2000.

CRUZ, Regina Célia Fernandes; FERNANDES, Helane de Fátima Gomes. Simbolismo sonoro do PB: o estudo dos ideofones. Revista de Estudos da Linguagem, v. 12, n. 2, p. 439-458, 2004.

CUNHA, Maria Angélica Furtado da; OLIVEIRA, Mariangela Rios de; MARTELOTTA, Mário Eduardo (Orgs.). Linguistica funcional: teoria e prática. Rio de Janeiro: DP\&A, 2003. 
CUSKLEY, Christine; KIRBY, Simon. Synaesthesia, cross-modality, and language evolution. In: Oxford bandbook of synaesthesia. Oxford University Press, 2013, p. 869-907.

CUSKLEY, Christine; SIMNER, Julia; KIRBY, Simon. Phonological and orthographic influences in the bouba-kiki effect. Psychological research. 2017, p.119-130.

FERNANDES, Helane de Fátima Gomes; CRUZ, Regina Célia Fernandes. Simbolismo sonoro, ideofones e iconicidade: expressividade sonora no português regional paraense e algumas exposições. In: JORNADA DE LETRAS: LÍNGUA, LITERATURA E OUTRAS LINGUAGENS, n.3, 2011, Cametá. Anais Lingua, literatura e outras linguagens. Cametá: Editora da Faculdade de Linguagem do Campus de Cametá, 2011. p. 184-216.

FORT, Mathilde; MARTIN, Alexander; PEPERKAMP, Sharon. Consonants are more important than vowels in the bouba-kiki effect. In: Language and Speech, 58(2), 2015, p. 247-266.

FURTADO DA CUNHA, Maria Angélica; TAVARES, Maria Alice. Linguística funcional e ensino de gramática. In: FURTADO DA CUNHA, Maria Angélica; TAVARES, Maria Alice (Orgs.) Funcionalismo e ensino de gramática. Natal: EDUFRN, 2007, p. 12-51.

GIVÓN, Talmy. Isomorphism in the grammatical code: cognitive and biological considerations. In: Studies in Language, Amsterdam, v. 15, n. 1, p. 85-114, 1991.

GIVÓN, Talmy. Functionalism and grammar. Amsterdam: John Benjamins, 1995.

HIRATA, Sachiko; UKITA, Jun; KITA, Shinichi. Implicit phonetic symbolism in voicing of consonants and visual lightness using Garner's speeded classification task. Percept. Mot. Skills 113, 929-940, 2011.

HOLLAND, Morris K.; WERTHEIMER, Michael. Some physiognomic aspects of naming, or, maluma and takete revisited. In: Perceptual and Motor Skills, v. 19, p.111-117, ago. 1964.

HOPPER, Paul J. Emergent grammar. In: TOMASELLO, Michael (Ed.) The new psychology of language: cognitive and functional approaches to language structure. New Jersey/ London: Lawrence Erlbaum, 1998. p. 155-175.

HOPPER, Paul J. Emergent grammar and temporality in interactional linguistics. In: AUER, Peter; PFÄNDER, Stefan (Eds.) Constructions: emerging and emergent. Berlin: De Gruyter, 2011. p. 22-44.

IMAI, Mutsumi; KITA, Sotaro; NAGUMO, Miho; OKADA, Hiroyuki. Sound symbolism facilitates early verb learning. In: Cognition, v. 109, p. 54-65, out. 2008.

IMAI, Mutsumi; KITA, Sotaro. The sound symbolism bootstrapping hypothesis for language acquisition and language evolution. In: Philosophical Transactions of the Royal Society B, v. 369, p. 1-13, set. 2014.

JAEGER, Tim Florian; TILY, Harry. On language "utility": Processing complexity and communicative efficiency. Wiley Interdisciplinary Reviews: Cognitive Science, v. 2, p. 323-335, mai/jun . 2011. 
KITA, Sotaro. Two-dimensional semantic analysis of Japanese mimetics. Linguistics, v. 35 (2), p. 379-415, 2017.

KITA, Sotaro; ÖZYÜREK, Asli; ALLEN, Shanley; ISHIZUKA, Tomoko. (2010). Relationship between iconic gestures and sound symbolic words i: Evidence for multimodal protolanguage [abstract]. In: Proceedings of the 8th International conference on the Evolution of Language (EVOLANG 8), Singapore: World Scientific, p. 429-431, 2010.

KÖHLER, Wolfgang. Gestalt psychology. Nova York: Liveright, 1929.

KÖHLER, Wolfgang. Gestalt psychology, 2 ed. Nova York: Liveright. 1947.

LOCKWOOD, Gwilym; DINGEMANSE, Mark. Iconicity in the lab: a review of behavioral, developmental, and neuroimaging research into sound-symbolism. In: Frontiers in Psychology, v. 6:1246, 2015.

LOCKWOOD, Gwilym; DINGEMANSE, Mark; HAGOORT, Peter. Sound-symbolism boosts novel word learning. In: Journal of Experimental Psychology: Learning, Memory, and Cognition, v. 42(8), p.1274-1281, 2016.

MATTOS E SILVA, Rosa Virgínia. Estruturas trecentistas: elementos para uma gramática do português arcaico. Lisboa: Imprensa Nacional-Casa da Moeda, 1989.

MAURER, Daphne; PATHMAN, Thanujeni; MONDLOCH, Catherine J. The shape of boubas: Sound-shape correspondences in toddlers and adults. In: Developmental Science, v. 9, 316-322, 2006.

MELO, Helane de Fátima Fernandes. Ideofones: um estudo no falar paraense. 120 fls. Dissertação (Mestrado) - Universidade Federal do Pará, Centro de Letras e Artes, Belém, 2007.

MOLLICA, Maria Cecília. Mecanismos de indeterminação do sistema preposicional. In: GROBE, Sybille; ZIMMERMANN, Klaus. (Eds.) "Substandard" e mudança no português do Brasil. Frankfurt am Main: TFM, 1998. p. 357-69.

MOOS, Anja; SMITH, Rachel; MILLER, Sam R.; SIMMONS, David R. Cross-modal associations in synaesthesia: vowel colours in the ear of the beholder. In: i-Perception, v. 5, p. 132 $142,2014$.

NYGAARD, Lynne C.; COOK Allison E.; NAMY, Laura L. Sound to meaning correspondences facilitate word learning. In: Cognition, v. 112, p. 181-186, jul. 2009.

NIELSEN, Alan Kirkland Staun; RENDALL, Drew. The Sound of Round: Evaluating the Sound-Symbolic Role of Consonants in the Classic Takete-Maluma Phenomenon. In: Canadian Journal of Experimental Psychology, v. 65:2, p.115-24, 2011.

OHTAKE, Yuka; HARYU, Etsuko. Investigation of the process underpinning vowel- size correspondence. In: Japanese Psychological Research, v. 55, p. 390-399, 2013.

OLIVEIRA, Mariangela Rios de; CEZARIO, Maria Maura. PCN à luz do funcionalismo linguístico. Revista Linguagem e Ensino, v. 10, n. 1, p. 87-108, 2007. 
ORTEGA, Gerardo; SÜMER, Beyza; ÖZYÜREK, Aslı. Type of Iconicity Matters in the Vocabulary Development of Signing Children. Developmental Psychology, v. 53, n. 1, p. 89-99, jan. 2017.

PEIFFER-SMADJA, Nathan. Exploring the bouba/kiki effect. A behavioral and fMRI study. 20092010. 50 fls. Dissertação (Master Recherche en Sciences Cognitives Master Thesis) - Université Paris Descartes, Paris. 2009-2010.

RAMACHANDRAN, Vilayanur S.; HUBBARD, Edward M. Synaesthesia-A window into perception, thought and language. In: Journal of Consciousness Studies, v. 8, n. 12, p. 3-34, 2001.

ROGERS, Susan K.; ROSS, Abraham S. A cross cultural test of the Maluma-Takete phenomenon. In: Perception, v. 4, n. 1, p. 105-106. 1975.

SAUSSURE, Ferdinand. Curso de Linguística geral, 22 ed. São Paulo: Cultrix, 2000.

SIMNER, Julia; CUSKLEY, Christine; KIRBY, Simon. What sound does that taste? Crossmodal mappings across gustation and audition. In: Perception, v.39, p. 553-569, 2010.

STYLES, Suzy; GAWNE, Lauren. When does maluma/takete fail? Two key failures and a metaanalysis suggest that phonology and phonotactics matter. In: i-Perception, v. 8(4), p. 1-17, 2017.

TARTE, Robert D.; BARRITT, Loren S. Phonetic symbolism in adult native speakers of English: three studies. In: Language and Speech, v.14, p. 158-168, apr-jun. 2017.

TARTE, Robert D. Phonetic symbolism in adult native speakers of Czech. In: Language and Speech, v. 17, p. 87-94, jan. 1974.

THOMPSON, Patrick; ESTES, Zachary. Sound symbolic naming of novel objects is a graded function. In: The Quarterly Journal of Experimental Psychology, v. 64, 2392-2404, 2011.

THOMPSON, Robin L.; VINSON, David P.; WOLL, Bencie; VIGLIOCCO, Gabriella. The road to language learning is iconic: evidence from British sign language. In: Psychological Science, v. 23, p. 1443-1448, 2012.

TUROMAN, Nora; STYLES, Suzy J. 2017 Glyph guessing for 'oo' and 'ee': spatial frequency information in sound symbolic matching for ancient and unfamiliar scripts. In: Royal Society open science, v. 4: 170882, p. 1-14. 2017.

WESTBURY, Chris. Implicit sound symbolism in lexical access: evidence from an interference task. In: Brain Lang, v. 93 (1), p. 10-19, abr. 2005.

Recebido em 20/12/2017

Aceito em 22/2/2018 